\title{
Systematic review estimating the burden of dementia in the WHO Southeast Asia Region using Bayesian and frequentist approaches
}

\author{
Adrienne N Poon ${ }^{1,2 *}$, \\ Yawen Xiang ${ }^{1 *}$, Yelena \\ Zavalishina $^{1 *}$, Shant \\ Ayanian², Christopher F \\ Aitken $^{3}$, Andrew C Procter ${ }^{4}$, \\ Igor Rudan', Kit Yee Chan ${ }^{1,5}$ \\ ${ }^{1}$ Centre for Global Health Research, \\ Usher Institute of Population Health \\ Sciences and Informatics, University \\ of Edinburgh, UK \\ ${ }^{2}$ Department of Medicine, School of \\ Medicine \& Health Sciences, George \\ Washington University; Washington, \\ District of Columbia, USA \\ ${ }^{3}$ Department of Economics, School \\ of Social Sciences, Heriot-Watt \\ University, Edinburgh, UK \\ ${ }^{4}$ Accenture Federal Services, \\ Arlington, Virginia, USA \\ ${ }^{5}$ Nossal Institute for Global Health, \\ Melbourne School of Population \\ and Global Health, University of \\ Melbourne, Australia \\ * Joint-first authorship.
}

\begin{abstract}
Background Rapid increase in life expectancy in low- and middle-income countries including the World Health Organization's Southeast Asia Region (SEAR) has resulted in an increase in the global burden of dementia, which is expected to become a leading cause of morbidity. Accurate burden estimates are key for informing policy and planning. Given the paucity of data, estimates were developed using both a Bayesian methodology and as well as a traditional frequentist approach to gain better insights into methodological approaches for disease burden estimates.
\end{abstract}

Methods Seven databases were searched for studies published between 20102018 regarding dementia prevalence in SEAR, generating 8 relevant articles. A random-effects model (REM) and a Bayesian normal-normal hierarchical model (NNHM) were used to obtain the pooled prevalence estimate of dementia for people aged 60 and above in SEAR. The latter model was also developed to estimate age-specific dementia prevalence. Using UN population estimates for SEAR, total and age-specific projections of the burden of dementia in 2015, 2020 and 2030 were calculated.

Results The prevalence of dementia in SEAR was found to be 3\% (95\% confidence interval $(\mathrm{CI})=2-6 \%$ ) in those above age 60 based on REM, and $3.1 \%$ (95\% credible interval $=1.5-5.0 \%$ ) based on the NNHM. The estimated prevalence varies with age, increasing from $1.6 \%$ (95\% credible interval $=0.8-2.5 \%$ ) in people aged $60-69$ to $12.4 \%$ (95\% credible interval $=5.6-20 \%$ ) in people above the age of 80 . The risk of developing dementia increased exponentially with age. The number of people living with dementia in SEAR in 2015 was estimated at 5.51 million (95\% credible interval $=2.66-8.82$ million, with projections of 6.66 million (95\% credible interval $=3.21-10.7$ ), in 2020 and 9.6 million (95\% credible interval $=4.62-15.36)$ in 2030 .

Conclusion The burden of dementia in SEAR is substantial and will continue to increase reapidly by 2030. The lack of research focusing on dementia in SEAR points to a significant under-recognition of this disease. The projected rise in dementia cases in the future should prompt urgent governmental response to address this growing public health issue. We also argue that given the overall paucity of data for the region, the Bayesian approach offers a promising methodology for improved estimates of disease prevalence and burden and should continue to be explored.

In 2015, an estimated 47 million people worldwide suffered from dementia, a major incapacitating syndrome defined by the progressive loss of cognitive ability and independent living beyond normal aging [1]. This figure is predicted to rise to 75 million by 2030, and 132 million by 2050, and has a bigger economic impact than cancer, heart disease and stroke combined [2]. In 2015, an estimated US\$818 
billion was spent on dementia ( $1.1 \%$ of global Gross Domestic Product), and that is expected to rise to US $\$ 2$ trillion by 2030 [1]. Due to their large, rapidly aging populations, low- and lower-middle-income countries (LMICs) are expected to bear an increasing majority of this burden, accounting for up to $71 \%$ of global dementia cases by 2050 [1]. The condition not only negatively affects the quality of life of people living with dementia (PWD), but also imposes significant financial, emotional and opportunity costs on their families and caregivers, and it strains social and health resources. Despite medical advancements and breakthroughs taking place in this century, there is currently no cure or disease-modifying treatment.

The World Health Organization's (WHO) Southeast Asia Region (SEAR), consisting mainly of LMICs, is the second most populated region of the world, with a quarter of its population (1.9 billion) [3]. There have only been three prior estimates that include dementia in the SEAR region, with the more recent covering estimates for the period of 1980-2009 [4-6]. Rapid economic, health and demographic transitions have occurred in this region since the last estimates were published, leading to a rise of non-communicable diseases including dementia. The evidence for dementia in the SEAR region has also been expanded over the last decade. Therefore, a separate and more comprehensive study of SEAR with updated estimates is urgently needed.

While disease burden estimates have traditionally relied on the frequentist analytic approach [7-9], Bayesian methods are growing in popularity in medical research (e.g., Prince 2013 [5]; Nichols 2019 [6]. The latter framework is considered more appropriate for meta-analyses with very few available studies because it allows additional information to be incorporated into current estimates $[7,8]$. That additional information, embedded in a prior, introduces a natural form of regularisation into the estimation procedure, helping to improve precision. Other, similar meta-analyses are ideal sources for that information: they represent credible distillations of available scientific evidence. With existing meta-analytic evidence forming the basis for the prior, the posterior produced by the Bayesian procedure can be interpreted as an updated summary. This in turn ensures consistency between current and prior estimates [9]. Two global health organizations that work on the global prevalence of dementia have both opted for Bayesian methods. The estimates produced by Prince and colleagues in 2013 assumed that the prevalence of dementia follows a gamma distribution [5]. The latest regional dementia estimate published in 2019 by the Institute for Health Metrics and Evaluation (IHME) used a Bayesian method [6]. An optimal approach, however, has not yet been determined and methods for Bayesian application to prevalence estimates is an area of ongoing study.

The overall aim of this study is to update the dementia prevalence estimates for SEAR with greater accuracy through utilizing two approaches to estimation: frequentist and Bayesian. We aim to achieve this by conducting a comprehensive systematic review of data that has emerged from the region since 2009 , when the previous estimates were published, using a greater number of academic databases. Given the paucity of data, we decided to use the Bayesian approach with confirmation by the traditional frequentist approach to generate more reliable estimates for disease burden. Generating appropriate models for Bayesian analysis may be challenging and thus we explored the use of the Bayesian Random-Effects Meta-Analysis (bayesmeta), a newer and simpler to operate statistical open source package in R. It is hoped that the updated estimates will help draw attention to the growing burden of dementia in SEAR as part of a global trend. This study can inform policy and planning, while methodological insights generated from the comparison of analytic approaches will guide development of future disease burden estimates.

\section{METHODS}

\section{Study selection}

We sought to include prospective population-based studies of dementia prevalence in countries in the WHO's SEAR published between 2010 and November 2018. The study was conducted and reported in accordance with the Preferred Reporting Items for Systematic Reviews and Meta-Analyses (PRISMA) guidelines [10] and involved a parallel systematic review conducted in May and November 2018 of studies on the epidemiology of dementia. Seven databases were searched: PubMed, MEDLINE, EMBASE, Global Health Library (CABI), Global Index Medicus, PsycInfo, and the BIOSIS Citation Index (Table S1 and Appendix S1 in the Online Supplementary Document).

1,556 studies were identified with 2 additional studies found through hand searches of reference lists. After the removal of duplicates, 1,216 articles remained of which 1,160 articles were further excluded based on the title relevance. 56 abstracts were then screened and 19 full-text articles were then analysed for inclusion/exclusion criteria, study design and the use of case definitions. The remaining 8 studies were 


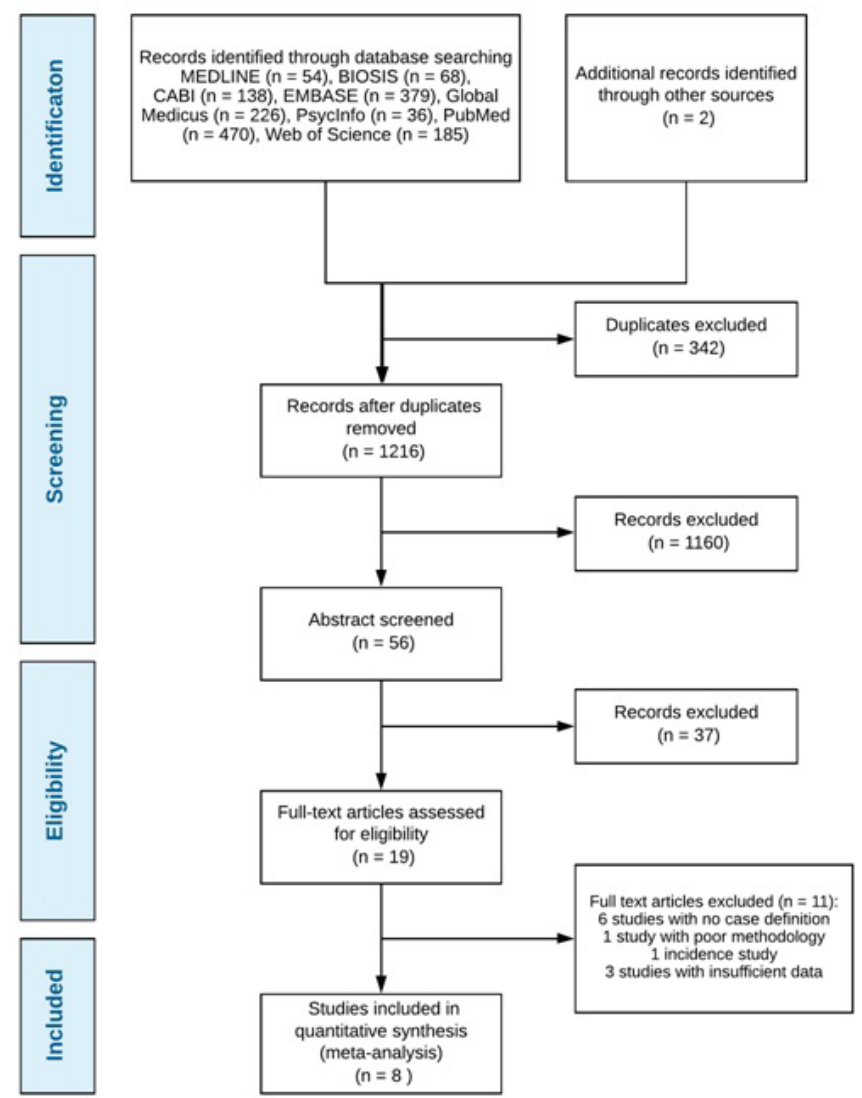

Figure 1. PRISMA flow diagram for selection of studies included in the systematic review. used for a meta-analysis [11-18]. See Figure 1 for the PRISMA diagram on study selection.

The included papers were critically appraised using the modified Joanna Briggs Institute (JBI) Critical Appraisal checklist for prevalence studies [5,19] (Table S2; Appendix S2 in the Online Supplementary Document). For each eligible study, we extracted the following data: country where the study was conducted, urban/rural setting, the period of study, sample size (denominator), number of dementia cases (numerator) and/or unweighted dementia prevalence. Wherever possible, data by age group, gender and types of dementia were also extracted.

\section{Study characteristics}

The 8 included studies took place in 2 countries of the SEAR - 7 from India [11-13,15-18] and 1 from Thailand [15]. Half of the studies were conducted in urban settings [11,16-18] while the other half were carried out in rural areas [12-15]. All studies took place between 2010 and 2017. Most studies adopted a two-stage design, which involved an initial screening by field workers and confirmation of dementia cases by a specialist. 2 out of 8 studies also included a third stage where researchers screen negative cases to identify false positives $[11,15]$. Most studies used a modified version of the Mini-Mental Status Exam (MMSE) as a screening tool. DSM-IV and ICD-10 were the most popular diagnostic tools used for case ascertainment. The study details can be found in Table 1 .

Table 1. Study details

\begin{tabular}{|c|c|c|c|c|c|c|}
\hline Study & Authors (Yeak) & Region/Country & Setting & Screening Tools & Outcome ascertainment & Study Design \\
\hline 1 & Banerjee (2017) [11] & Kolkata, India & Urban & KBSB & $\begin{array}{l}\text { DSM-IV; } \\
\text { NINCDS-ADRDA; } \\
\text { NINCDS-AIREN }\end{array}$ & $\begin{array}{l}\text { 3-stage design } \\
\text { cross-sectional study }\end{array}$ \\
\hline 2 & Gurukartick (2016) [12] & $\begin{array}{l}\text { Thiruvennainallur in } \\
\text { Villupuram District of Tamil } \\
\text { Nadu, India }\end{array}$ & Rural & VSID & DSM-IV & $\begin{array}{l}\text { 2-stage design } \\
\text { cross-sectional study } \dagger\end{array}$ \\
\hline 3 & Gambhir (2014) [13] & $\begin{array}{l}\text { Chiraigaon block of Varanasi } \\
\text { District, India }\end{array}$ & Rural & HMSE & $\begin{array}{l}\text { DSM-IV-TR; } \\
\text { ICD-10 }\end{array}$ & $\begin{array}{l}2 \text {-stage design } \\
\text { cross-sectional study } \dagger\end{array}$ \\
\hline 4 & Senanarong (2013) [14] & Siriraj, Thailand & Rural & TMSE & DSM-IV & $\begin{array}{l}2 \text {-stage design } \\
\text { cross-sectional study } \dagger\end{array}$ \\
\hline 5 & Tiwari (2013) [15] & Luchnow, India & Rural & $\begin{array}{l}\text { HMSE; } \\
\text { CAMDEX-R }\end{array}$ & DSM-IV; ICD-10 & $\begin{array}{l}\text { 3-stage design } \\
\text { cross-sectional study* }\end{array}$ \\
\hline 6 & Seby (2011) [16] & $\begin{array}{l}\text { Pune district of Maharashtra } \\
\text { State, India }\end{array}$ & Urban & $\begin{array}{l}\text { GHQ-12; } \\
\text { MMSE }\end{array}$ & ICD-10 & $\begin{array}{l}2 \text {-stage design } \\
\text { cross-sectional study } \dagger\end{array}$ \\
\hline 7 & Mathuranath (2010) [17] & $\begin{array}{l}\text { Trivandrum, Kerala State, } \\
\text { India }\end{array}$ & Urban & ACE; MMSE & $\begin{array}{l}\text { DSM-IV; NINCDS- } \\
\text { ADRDA; Hachins- } \\
\text { ki's Ischemic Scale }\end{array}$ & $\begin{array}{l}\text { 2-stage design } \\
\text { cross-sectional study } \dagger\end{array}$ \\
\hline 8 & Saldanha (2010) [18] & $\begin{array}{l}\text { Pune and Kirkee canton- } \\
\text { ments, Maharashtra, India }\end{array}$ & Urban & MMSE; CSI-D & ICD-10 & $\begin{array}{l}\text { Single phase } \\
\text { cross-sectional survey }\end{array}$ \\
\hline
\end{tabular}

KCSB - Kolkata Cognitive Screening Battery, VSID - Vellore Screening Instrument for Dementia, HMSE - Hindi Mini Mental state examination, TMSE Thai Mental State Examination, CAMDEX-R - Cambridge Examination for Mental Disorders of the Elderly - Revised (CAMDEX-R), GHQ-12 - General Health Questionnaire-12, MMSE - Mini Mental State Examination, ACE - Addenbrooke's Cognition Examination, CSI-D - 10/66 Research Group Community Screening Instrument for Dementia, ICD - International Classification of Diseases, DSM - Diagnostic and Statistical Manual of Mental Disorders, NINDS-AIREN - National Institute of Neurological Disorders and Stroke Association criteria for vascular dementia, NINCDS-ADRDA - National Institute of Neurological and Communicative Disorders and Stroke and the Alzheimer's Disease and Related Disorders Association criteria for Alzheimer's disease *3-stage design cross-sectional study: 1. Screening by trained fieldworkers; 2 . Confirmation of suspected cases by consultant psychiatrists/ psychiatric team; 3. Checking of unsuspected cases by consult psychiatrists/ psychiatric team for false negatives.

$\uparrow 2$-stage design cross-sectional study: 1 . Screening by trained fieldworkers; 2 . Confirmation of suspected cases by consultant psychiatrists/ psychiatric team. 
The total sample size for the 8 studies combined was 28543 participants. There was considerable variation in the number of participants between studies, ranging from 202 to 17584 (Table 2). The median study sample size was 2072. All studies included individuals aged 50 years and above, and the most common classifications of age groups were age 60 years and above or age 65 years and above. Two studies recruited a regionally representative sample by taking into account the cultural and socioeconomic background of the participants [11-17]. The remaining studies used representative samples of the rural or urban community dwelling elderly populations. The proportion of female participants was around the same as male participants.

Tahle 2. Detailed sampling characteristics

\begin{tabular}{|c|c|c|c|c|c|}
\hline Study & Authors (YeAR) & Sample Selection & Participant recruitment & SaMPIE SIZE AND RESPONSE RAIE & Participants traits \\
\hline \multirow[t]{2}{*}{1} & \multirow[t]{2}{*}{ Banerjee (2017) [11] } & \multirow{2}{*}{$\begin{array}{l}\text { Representative of the re- } \\
\text { gion in terms of socioeco- } \\
\text { nomic and cultural levels }\end{array}$} & \multirow[t]{2}{*}{$\begin{array}{l}\text { Stratified and ran- } \\
\text { dom sampling }\end{array}$} & $\begin{array}{l}100802 \text { approached and } \\
\text { analysed }\end{array}$ & \multirow[t]{2}{*}{$\begin{array}{l}47.2 \% \text { female, } \geq 50 \\
\text { years old }\end{array}$} \\
\hline & & & & Attrition <1\% & \\
\hline \multirow[t]{2}{*}{2} & \multirow[t]{2}{*}{ Gurukartick (2016) [12] } & \multirow{2}{*}{$\begin{array}{l}\text { Rural community dwelling } \\
\text { elderly population }\end{array}$} & \multirow{2}{*}{$\begin{array}{l}\text { Random and pro- } \\
\text { portional sampling }\end{array}$} & 1304 analysed & \multirow{2}{*}{$\begin{array}{l}44.9 \% \text { female, } \geq 65 \\
\text { years old }\end{array}$} \\
\hline & & & & $\begin{array}{l}\text { Sample size calculation } \\
\geq 1300\end{array}$ & \\
\hline \multirow[t]{2}{*}{3} & \multirow[t]{2}{*}{ Gambhir (2014) [13] } & \multirow{2}{*}{$\begin{array}{l}\text { Rural community dwelling } \\
\text { elderly population }\end{array}$} & \multirow[t]{2}{*}{ Random sampling } & 728 analysed & \multirow{2}{*}{$\begin{array}{l}64.4 \% \text { female, } \geq 60 \\
\text { years old }\end{array}$} \\
\hline & & & & $54-80 \%$ for female & \\
\hline \multirow[t]{2}{*}{4} & \multirow[t]{2}{*}{ Senanorong (2013) [14] } & \multirow[t]{2}{*}{$\begin{array}{l}\text { Rural community dwelling } \\
\text { elderly population }\end{array}$} & \multirow{2}{*}{$\begin{array}{l}\text { Catchment from } \\
\text { primary care unit of } \\
\text { Siriraj Hospital }\end{array}$} & $\begin{array}{l}1998 \text { approached, } 1973 \\
\text { analysed (98.7\%) }\end{array}$ & \multirow[t]{2}{*}{$\begin{array}{l}65.1 \% \text { female, } \geq 60 \\
\text { years old }\end{array}$} \\
\hline & & & & $\begin{array}{l}\text { Sample size calculation } \\
\geq 1948\end{array}$ & \\
\hline \multirow[t]{2}{*}{5} & \multirow[t]{2}{*}{ Tiwari (2013) [15] } & \multirow[t]{2}{*}{$\begin{array}{l}\text { Rural community dwelling } \\
\text { elderly population }\end{array}$} & \multirow[t]{2}{*}{ Random sampling } & $\begin{array}{l}2324 \text { approached, } 2146 \\
\text { analysed }(92.3 \%)\end{array}$ & \multirow[t]{2}{*}{$\begin{array}{l}52.6 \% \text { female, } \geq 60 \\
\text { years old }\end{array}$} \\
\hline & & & & $\begin{array}{l}\text { Sample size calculation } \\
\geq 2060\end{array}$ & \\
\hline 6 & Seby (2011) [16] & $\begin{array}{l}\text { Urban community dwell- } \\
\text { ing elderly population }\end{array}$ & $\begin{array}{l}\text { Consecutive sam- } \\
\text { pling }\end{array}$ & $\begin{array}{l}218 \text { approached, } 202 \\
\text { analysed (92.7\%) }\end{array}$ & $\begin{array}{l}49.1 \% \text { female, } \geq 65 \\
\text { years old }\end{array}$ \\
\hline 7 & Mathuranath (2010) [17] & $\begin{array}{l}\text { Representative of the re- } \\
\text { gion in terms of socioeco- } \\
\text { nomic and cultural levels }\end{array}$ & $\begin{array}{l}\text { Door to door sur- } \\
\text { vey }\end{array}$ & $\begin{array}{l}2690 \text { eligible, } 2446 \text { ana- } \\
\text { lysed (90.9\%) }\end{array}$ & $\begin{array}{l}59.4 \% \text { female, } \geq 55 \\
\text { years old }\end{array}$ \\
\hline 8 & Saldanha (2010) [18] & $\begin{array}{l}\text { Community dwelling pop- } \\
\text { ulation }\end{array}$ & $\begin{array}{l}\text { Random sampling } \\
\text { then door to door } \\
\text { survey }\end{array}$ & $\begin{array}{l}2145 \text { approached, } 2119 \\
\text { analysed, (98.8\%) }\end{array}$ & $\begin{array}{l}60.5 \% \text { female, } \geq 65 \\
\text { years old }\end{array}$ \\
\hline
\end{tabular}

\section{Quality assessment}

There was considerable variation in the quality of the included studies (Appendix S2 in the Online Supplementary Document). The quality score ranged from 12 to 17 out of 18 . Out of 8 studies, 2 study samples were representative of the target population $[11,17]$ and 3 were sampled in an unbiased manner $[11,14,17]$. In general, sample sizes were appropriate, and 3 studies carried out a sample size calculation prior to recruitment $[12,14,15]$. Most studies clearly documented exclusion criteria, the number of refusals and loss to follow-up. Response rates were high overall: 6 had a response rate over 90\% [11,1418]. All studies used a well-recognized diagnostic manual and conducted different tests to exclude other conditions with similar clinical manifestations. For instance, all studies made effort to exclude depression as a differential diagnosis.

\section{Data analysis}

Given the paucity of data, dementia prevalence was estimated using a Bayesian approach and confirmed by a frequentist approach. This allows for comparison of the validity of estimates particularly given the limitations in data availability and the opportunity to explore the utility of using a simpler statistical package to generate a normal-normal hierarchical model (NNHM) for disease burden estimates with limited data. All analyses were conducted using the statistical software R (version 3.5.2, R Core Team, Vienna, Austria).

\section{Bayesian approach}

A Bayesian approach allows for estimates based on smaller numbers of studies by allowing prior estimates to be incorporated, which improves precision [7-9]. For instance, the 2009 estimate of 6.38\% demen- 
tia prevalence in SEAR for people age $\geq 60$ [4] indicates that the 2020 prevalence will probably be under $10 \%$. If an updated meta-analysis included studies that were methodologically flawed or were based on a highly biased sample that produced an unrealistic prevalence (eg, >25\%), the outlier effect would be restrained due to prior knowledge [9].

The interpretation of results generated by a Bayesian approach is also more intuitive. In the frequentist analysis, the concept of confidence interval is commonly misunderstood [20]. A 95\% confidence interval (CI) means that if we were to take repeated samples from the population, and calculate the confidence intervals each time, then it would be expected that $95 \%$ of the calculated confidence intervals would be such as to include the true population parameter [21]. 95\% credible interval produced from the Bayesian analysis, on the other hand, means that given the observed data, there is a $95 \%$ probability that the true value of prevalence falls within the credible interval [20]. This means that the credible intervals produced are much more relevant in global health research.

The age-specific prevalence was pooled using the bayesmeta package of R [20]. The Bayesian method can allow us to update the current state of knowledge by considering the newly extracted data alongside previously published literature. In 2009, Alzheimer's Disease International (ADI) published the age-specific prevalence of dementia for different world regions [4]. The age-specific prevalence of dementia in South Asia published by ADI was used to inform the specification of the prior for the Bayesian approach. Importantly, the ADI's analysis was based on studies that are entirely distinct from those included in this work. Only studies with age-specific prevalence data were included in the meta-analysis. A Bayesian normal-normal hierarchical model (NNHM) was constructed with age groups as the independent variable and age-specific prevalence as the dependent variable. The NNHM model was chosen because it mirrors many of the key distributional assumptions embedded within the frequentist random-effects model $[20,22]$.

The number of participants screened and the number of PWD identified from each study were first sorted into 10-year age group bins. Participants over the age of 80 were all allocated into an "over 80 " bin. Previous research has held that dementia is rare among younger individuals and the prevalence of the disease increases with age. However, the evidence on the rate itself is mixed, particularly for older cohorts. To reflect this pattern of uncertainty, we set the prior variance to increase with age: for age groups 60-69, $70-79$ and over 80 , it was $0.092,0.152$ and 0.32 respectively. The prevalence estimates for each of these groups were pooled, and $95 \%$ credible intervals were obtained.

As a sensitivity test for the baysmeta package, Just another Gibbs sampler (JAGS) was used to construct a more traditional model simulated via Markov chain Monte Carlo (MCMC) methods, which produced estimates of disease burden for each age group bin highlighted above using a similar NNHM model. JAGS is an open source algorithm used often in Bayesian analysis to simulate draws from target posterior distributions [23].

\section{Frequentist approach}

Crude prevalence estimates were pooled using a frequentist approach with the metafor package of R [24]. Given the available evidence, which strongly indicates a degree of heterogeneity in prevalence rates within the region, a random effects model was thought most appropriate. Note the subtle point that we are interested in conducting inference about the SEAR dementia prevalence rate in general - that is, unconditional inference in the language of [25]. Again, this suggests the REM is the most suitable frequentist model to adopt. All studies with crude prevalence estimates were included in the analysis.

To assess heterogeneity between studies, Cochrane's $Q$ test, I-squared $\left(\mathrm{I}^{2}\right)$ statistics and tau-squared $\left(\mathrm{T}^{2}\right)$ statistics were examined. Cochrane's Q test tests the null hypothesis that the true prevalence is the same in all primary studies included in the meta-analysis. A p-value of less than 0.05 shows evidence to reject the null hypothesis and indicates the presence of statistical heterogeneity among the included studies [26]. The $\mathrm{I}^{2}$ statistic represents the percentage of total variation across studies that is due to true heterogeneity instead of chance. Generally, $\mathrm{I}^{2}$ values of 25\%,50\% and 75\% are interpreted as low, moderate and high heterogeneity [27]. $\mathrm{T}^{2}$ is the variance of the prevalence parameter across the population of studies and reflects, again, heterogeneity in the true prevalence rate [28].

\section{Burden estimation}

The number of PWD in 2015, 2020 and 2030 was estimated by multiplying the prevalence obtained by both methods with the number of people from SEAR in 2015, 2020 and 2030 using data from the UN 
Population Division [29]. The burden of dementia in each age group was only estimated with the Bayesian model and is calculated by multiplying the age-specific prevalence and the number of people in the corresponding age groups in 2015, 2020 and 2030. Note these projections are made on a ceteris paribus basis: they allow only for changes to the population size; the prevalence rate and other key parameters are assumed to be constant through time.

\section{RESULTS}

\section{Prevalence estimates}

Five studies reported age-specific prevalence of dementia [12,14,17-19] and were included in the Bayesian NNHM. For people aged 60 and above, dementia prevalence was found to be 3.1\% (95\% credible interval $=1.5-5.0 \%)$. The prevalence of dementia ranged from $1.6 \%(95 \%$ credible interval $=0.8-2.5 \%)$ in those between 60-69 years of age to $12.4 \%$ (95\% credible interval $=5.6-20 \%$ ) in those above the age of 80 (Table 3). As a test of the sensitivity of the results, we conducted the analysis using a modified NNHM prior setup and estimated the model via classical MCMC methods: with that, we obtained similar results (Appendix S3 in the Online Supplementary Document).

All 8 studies reported overall dementia prevalence and were pooled using REM (Figure 2). Based on the frequentist model, the unadjusted crude prevalence for people over 60 years was estimated to be $3.0 \%$ (95\% CI $=2-6 \%)$. Cochrane's Q test revealed that there was significant heterogeneity among the included studies. True heterogeneity, indicated by $\mathrm{I}^{2}$, was above $90 \%$, which was very high. The variance of prevalence parameter across the study populations, or $\mathrm{T}^{2}$, was 0.82 .

\section{Burden estimates}

Overall, there were 175.7 million people aged 60 and above in SEAR in 2015. This number is projected to increase to 209.2 million in 2020 and 292.7 million in 2030 [29]. Based on the results obtained with the Bayesian NNHM model, we estimate that the number of PWD will increase from 5.51 million (95\% credible interval $=2.66-8.82$ million) in 2015 to 6.66 million ( $95 \%$ credible interval $=3.21-10.7$ million) in 2020. In 2030, it is projected that there will be nearly 10 million (9.60 million, 95\% credible inter$\mathrm{val}=4.62-15.36$ million) PWD in SEAR (Figure 3, Table 4). Similarly, based on the REM, we estimate there were 5.21 million (95\% CI= 3.47 - 10.40 million) PWD in SEAR in 2015 (Table 4). The number

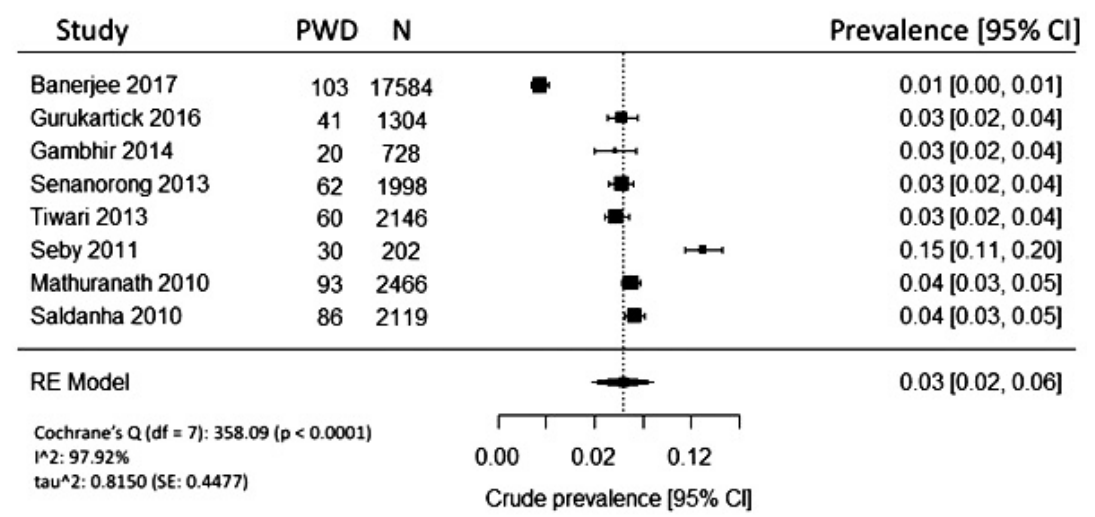

Figure 2. Crude prevalence for individuals over age 60.

Table 3. Prevalence of dementia in 10-year age groups in SEAR

\begin{tabular}{|c|c|c|c|c|}
\hline Age & $\begin{array}{l}\text { PoOLED PREVALENCE ESTIMATE (95\% } \\
\text { CREDIBLE INTERVAL) }\end{array}$ & $\begin{array}{l}\text { Number of PWD in SEAR in } 2015 \\
\text { (thousands) }\end{array}$ & $\begin{array}{l}\text { Projected nUMber of PWD IN SEAR IN } \\
2020 \text { (thousands) }\end{array}$ & $\begin{array}{l}\text { Projected nUMBer of PWD in SEAR in } \\
2030 \text { (thousands) }\end{array}$ \\
\hline $60-69$ & $0.016(0.008-0.025)$ & $1691.90(845.95-2,643.60)$ & $2063.18(1031.59-3223.73)$ & 2733.55 (1366.78-4271.18) \\
\hline $70-79$ & $0.034(0.017-0.055)$ & $1739.41(869.70-2,813.75)$ & $2,021.50(1010.75-3270.08)$ & $3115.76(1557.88-5040.20)$ \\
\hline$\geq 80$ & $0.124(0.056-0.200)$ & $2082.46(940.46-3,358.80)$ & $2579.70(1165.02-4160.80)$ & $3747.65(1692.49-6044.60)$ \\
\hline$\geq 60$ & $0.0314(0.015-0.050)$ & $5513.77(2656.12-8816.15)$ & $6664.38(3207.37-10654.61)$ & $9596.96(4,617.14-15355.98)$ \\
\hline
\end{tabular}

PWD - people with dementia, SEAR - Southeast Asia Region 


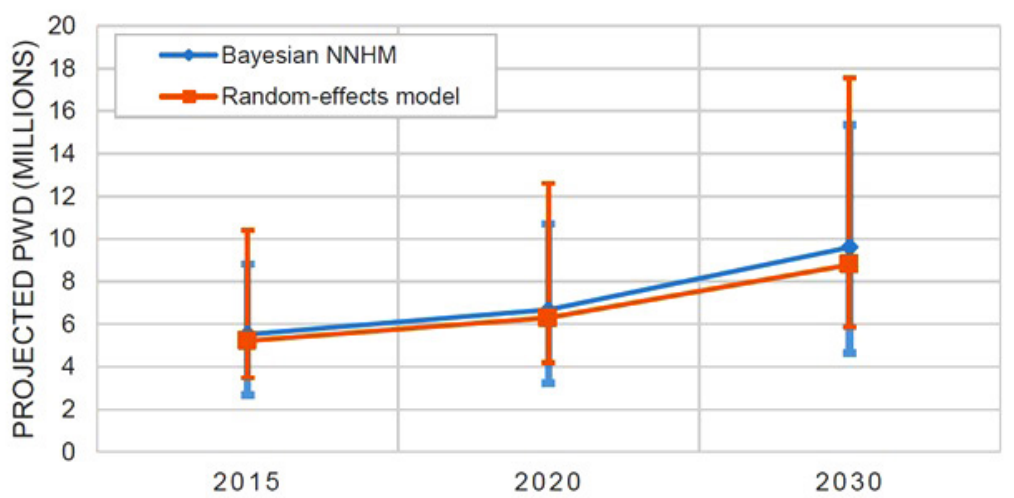

Figure 3. Projected dementia cases in SEAR by Bayesian and frequentist models. Note: Due to the conceptual differences between the analytic approaches we would like to remind the reader that the $95 \%$ confidence intervals (Random-effects model) and 95\% credible intervals (Bayesian) cannot be interpreted interchangeably.

Table 4. Burden estimate comparisons between Bayesian and frequentist models

\begin{tabular}{lcc} 
Year & Bayesian NNHM & RandOM-EFFEctS MODEl \\
2015 & $5.51(2.66-8.82)$ million* & $5.21(3.47-10.40)$ million \\
\hline 2020 & $6.66(3.21-10.7)$ million & $6.28(4.18-12.60)$ million \\
\hline 2030 & $9.60(4.62-15.36)$ million & $8.78(5.85-17.56)$ million \\
\hline \multicolumn{2}{l}{ NNHM - normal-normal hierarchical model }
\end{tabular}

lion ( $95 \%$ credible interval $=1.17-4.16$ million) in 2020 and 3.75 million (95\% credible interval $=1.69$ 6.04 million) in 2030 .

\section{DISCUSSION}

We aimed to estimate the prevalence of dementia in the WHO's SEAR and adopted both Bayesian and traditional frequentist approaches to optimize our understanding of the burden of dementia within this region. We searched for studies published in the English language between 2010 and 2018 and ultimately found 8 for inclusion, 7 of which were from India, and 1 was conducted in Thailand. The Bayesian approach may be more useful in regions with limited data availability. We therefore compared it to the traditional frequentist approach, which is more commonly used in studies with larger amounts of data available. Our Bayesian estimates revealed that there were about 5.51 million people living with dementia in SEAR in 2015, consistent with the most recent estimates produced by IHME, which found that there were 5.47 million people with dementia in SEAR in 2016 [6].

Despite our best efforts to estimate dementia prevalence in Southeast Asia, current epidemiological knowledge of dementia prevalence in this region proved to be sparse. Out of the eleven countries in the area, only India and Thailand provided studies with sufficient data. Indonesia and Bangladesh are the third most populated countries in Southeast Asia after India, which may be most affected by dementia in the upcoming years, yet lack of published studies makes it extremely difficult to assess and predict the impact dementia will have on these countries. With an estimated 5.51 million already living with dementia and close to 10 million projected to be affected by 2030, urgent public health action is necessary.

The crude prevalence of dementia in people aged 60 years and above was 3.0\% (95\% CI $=2-6 \%$ ) based on all included studies. The age-adjusted prevalence of dementia was $3.1 \%$ (95\% credible interval $=1.5-$ $5.0 \%)$. As the majority of the included studies in this current review came from India, the most comparable (external) prevalence estimate was an estimate produced by the Alzheimer's and Related Disorders Society of India in 2010. They found that the prevalence of dementia in India ranged from $0.6 \%$ to $3.5 \%$ in rural areas and $0.9 \%$ to $4.8 \%$ in urban areas [30]. Our estimate, therefore, is consistent with the findings of current literature. This review also supports the long established relationship between age and prevalence of dementia. The prevalence ranged from 1.6\% in the 60-69 age group to $12.4 \%$ in the above 80 age group. In comparison, sensitivity analysis using Bayesian approach with the JAGS sampler 
showed similar though slightly higher prevalence estimates with $2.3 \%$ for ages $60-69,4.9 \%$ for ages 70 $79,13.5 \%$ for above age 80 , respectively.

The Delphi Consensus reported prevalence of dementia for two regions in Southeast Asia - SEAR B which includes Indonesia, Thailand and Sri Lanka, and SEAR D which is comprised of India and South Asian countries. Their estimates for SEAR D which include India and are most comparable to this research show that the prevalence of dementia varied between $0.4 \%$ in those between $60-64$ to $14 \%$ in people above 85 . However, it is more difficult to make comparisons of current estimates to the SEAR B region, as this review did not include any study from those regions in the data analysis. A more recent meta-analysis conducted by Prince et al in 2013 included five studies that showed how dementia prevalence in the South Asia region (most comparable to this review as it included India) for individuals over age 60 was 5.8\% [5].

Given that an optimal approach to estimating disease burden continues to be an area of research, the results lend validity to the use of the Bayesian NNHM approach as adopted in this review and support its further use. Future research is needed to explore the role of varying statistical approaches in prevalence and burden of disease estimates, but the results of this paper are promising, particularly in settings with limited data availability as in this review. Furthermore, the ability to use a user-friendly open source software to run complex full Bayesian analysis like bayesmeta allows for easier incorporation of this approach in future studies and allows greater accessibility to estimate burden of disease for researchers across the globe.

The use of PRISMA guidelines, careful selection of studies, and the use of validated quality assessment tools to ensure research rigour were strengths of this review. However, despite efforts to provide the best possible estimate of the prevalence of dementia in SEAR, there were some limitations. First, while the studies were carefully selected and chosen based on the quality of conducted research, the sample size of each study was relatively small compared to the overall population of the respective countries. Second, the estimates of the current study were based on only 2 of the 11 countries in the SEAR (mainly India, and to a lesser extent Thailand). The paucity of data from the rest of the region represents a serious limitation of our work. Third, the other countries in this region all have very distinct cultures and levels of development, which would plausibly lead to variation in their citizens' level of dementia risk. Fourth, the articles were restricted to English language only, potentially limiting the research from countries which may not have a large number of English publications. Fifth, some studies only included participants that speak Hindi, Thai or English, possibly underestimating the true number of participants suffering from dementia as many may be excluded for not speaking these languages. Sixth, sex-and age-specific prevalence of dementia could not be estimated due to lack of reporting from the included studies. Seventh, very few studies reported information on specific dementia types, making it difficult to estimate the prevalence of dementia subtypes (eg, Alzheimer's disease, vascular dementia). Lastly, it is worth underscoring that our estimates of the burden of dementia crucially relied on a number of strong assumptions: the prevalence remains the same and the population structure progresses as predicted by the UN estimates. It is likely that these will not be borne out. Nevertheless, the estimates give researchers and policymakers important and relevant insight into the future trend of dementia burden.

Dementia has been significantly underrecognized and underestimated in SEAR. This review estimated the prevalence of dementia in SEAR and showed that the number of people living with dementia in this region is substantial and is expected to rise, with over 10 million people projected to have dementia in this region by 2030. In 2015, the total costs of dementia in Southeast Asia reached US $\$ 7.3$ billion, which was around one third of Nepal's national GDP [1,31]. With the projected increase in dementia prevalence, the costs incurred will place a significant financial toll on governments, families and individuals.

There is an urgent need for more epidemiological research on dementia burden in SEAR. Nine out of eleven countries in SEAR have no published information on the prevalence of dementia. For future epidemiological studies, there should be greater emphasis on reporting of research findings. Apart from the crude dementia prevalence, all studies should aim to report on age-specific and sex-and-age-specific dementia prevalence. If resources allow, researchers should also attempt to estimate the prevalence of dementia subtypes. This information will be very useful for more accurate burden estimation and healthcare planning.

\section{CONCLUSION}

This review calls for greater recognition of dementia as a health priority and urges countries in SEAR to reassess their approach to addressing dementia. We found a significant burden of dementia in SEAR that is projected to nearly double by 2030. Governments should focus on promoting awareness of the condi- 
tion and giving recognition to the fact that dementia is not just a by-product of aging, but a recognized illness that has broad effects on the healthcare system and economy.

We demonstrated that the Bayesian approach offers benefits beyond the traditional frequentist approach to estimate disease prevalence and burden, particularly with significantly limited data. We were also able to demonstrate that the bayesmeta package running an NNHM model can offer similar estimates to the Bayesian JAGS algorithm. Further research is needed to assess if there is an optimal approach for disease burden estimates. The Bayesian approach offers a promising methodology for improved estimates and should continue to be explored, especially since accessible open source software for this method is now available.

Future research should focus on targeting risk factors for dementia in SEAR as well as possible prevention measures. These future directions will help address the needs of and bring tailored interventions to various types of communities affected by dementia in these countries, with the long-term goal of generating greater understanding and policies to decrease the prevalence of this disabling disorder.

\footnotetext{
Funding: None.

Authorship contributions: KC, YZ, YX, ANP contributed towards conceptualizing this manuscript. YX, CF, and SA contributed to data analysis. AP and IR gave intellectual input. All authors contributed to writing and review of this manuscript.

Competing interests: The authors completed the ICMJE Unified Competing Interest form (available upon request from the corresponding author), and declare no conflicts of interest. Igor Rudan is the Co-Editor in Chief of the Journal of Global Health. To ensure that any possible conflict of interest relevant to the journal has been addressed, this article was reviewed according to best practice guidelines of international editorial organisations.

Additional material

Online Supplementary Document
}

1 Prince M, Wimo A. World Alzheimer Report 2015 - The Global Impact of Dementia. Alzheimer's Disease International. 2015. Available: https://www.alz.co.uk/research/WorldAlzheimerReport2015.pdf. Accessed: 28 September 2019.

2 Wimo A, Prince M. World Alzheimer Report 2010 - The Global Economic Impact of Dementia. Alzheimer's Disease International. 2010. Available: https://www.alz.co.uk/research/files/WorldAlzheimerReport2010.pdf. Accessed: 28 September 2019.

3 World Health Organization. History of the WHO South-East Asia Region. 2019. Available: http://www.searo.who.int/ about/history/en/. Accessed: 28 September 2019.

4 Prince M, Jackson J. World Alzheimer Report 2009. Alzheimer's Disease International. 2009. Available: https://www.alz. co.uk/research/files/WorldAlzheimerReport.pdf. Accessed: 28 September 2019.

5 Prince M, Bryce R, Albanese E, Wimo A, Ribeiro W, Ferri CP. The global prevalence of dementia: A systematic review and metaanalysis. Alzheimers Dement. 2013;9:63-75.e2. Medline:23305823 doi:10.1016/j.jalz.2012.11.007

6 GBD 2016 Dementia Collaborators. Global, regional, and national burden of Alzheimer's disease and other dementias, 1990-2016: a systematic analysis for the Global Burden of Disease Study 2016. Lancet Neurol. 2019;18:88-106. Medline:30497964 doi:10.1016/S1474-4422(18)30403-4

7 Seide SE, Röver C, Friede T. Likelihood-based random-effects meta-analysis with few studies: empirical and simulation studies. BMC Med Res Methodol. 2019;19:16. Medline:30634920 doi:10.1186/s12874-018-0618-3

8 Higgins JPT, Thompson SG, Spiegelhalter DJ. A re-evaluation of random-effects meta-analysis. J R Stat Soc Ser A Stat Soc. 2009;172:137-59. Medline:19381330 doi:10.1111/j.1467-985X.2008.00552.x

9 Spiegelhalter DJ. Incorporating Bayesian ideas into health-care evaluation. Stat Sci. 2004;19:156-74. doi:10.1214/088342304000000080

10 Moher D, Liberati A, Tetzlaff J, Altman DG, Altman D, Antes G, et al. Preferred reporting items for systematic reviews and meta-analyses: The PRISMA statement. PLoS Med. 2009;6:e1000097. Medline:19621072 doi:10.1371/journal. pmed. 1000097

11 Banerjee TK, Dutta S, Das S, Ghosal M, Ray BK, Biswas A, et al. Epidemiology of dementia and its burden in the city of Kolkata, India. Int J Geriatr Psychiatry. 2017;32:605-14. Medline:27251089 doi:10.1002/gps.4499

12 Gurukartick J, Dongre AR, Shah D. Social determinants of dementia and caregivers' perspectives in the field practice villages of rural health training centre, Thiruvennainallur. Indian J Palliat Care. 2016;22:25-32. Medline:26962277 doi:10.4103/0973-1075.173952

13 Gambhir IS, Khurana V, Kishore D, Sinha AK, Mohapatra SC. A clinico-epidemiological study of cognitive function status of community-dwelling elderly. Indian J Psychiatry. 2014;56:365-70. Medline:25568477 
14 Senanarong V, Harnphadungkit K, Poungvarin N, Vannasaeng S, Chongwisal S, Chakorn T, et al. The dementia and disability project in Thai elderly: Rational, design, methodology and early results. BMC Neurol. 2013;13:3. Medline:23305293 doi:10.1186/1471-2377-13-3

15 Tiwari SC, Srivastava G, Tripathi RK, Pandey NM, Agarwal GG, Pandey S, et al. Prevalence of psychiatric morbidity amongst the community dwelling rural older adults in northern India. Indian J Med Res. 2013;138:504-14. Medline: 24434257

16 Seby K, Chaudhury S, Chakraborty R. Prevalence of psychiatric and physical morbidity in an urban geriatric population. Indian J Psychiatry. 2011;53:121-7. Medline:21772643 doi:10.4103/0019-5545.82535

17 Mathuranath PS, Cherian PJ, Mathew R, Kumar S, George A, Alexander A, et al. Dementia in Kerala, South India: prevalence and influence of age, education and gender. Int J Geriatr Psychiatry. 2010;25:290-7. Medline:19621355 doi:10.1002/gps.2338

18 Saldanha D, Mani MR, Srivastava K, Goyal S, Bhattacharya D. An epidemiological study of dementia under the aegis of mental health program, Maharashtra, Pune chapter. Indian J Psychiatry. 2010;52:131-9. Medline:20838500 doi:10.4103/0019-5545.64588

19 Munn Z. MClinSc SM, Lisy K, Riitano D, Tufanaru C. Methodological guidance for systematic reviews of observational epidemiological studies reporting prevalence and cumulative incidence data. Int J Evid-Based Healthc. 2015;13:147-53. Medline:26317388 doi:10.1097/XEB.0000000000000054

20 Röver C. Bayesian random-effects meta-analysis using the bayesmeta r package. J Stat Softw. 2020;93:1-51. doi:10.18637/ jss.v093.i06

21 Kirkwood BR, Sterne JAC. Essential Medical Statistics. Medical statistics. Hoboken: Wiley-Blackwell. 2003.

22 Rubin DB. Estimation in parallel randomized experiments. J Educ Stat. 1981;6:377-401. doi:10.3102/10769986006004377

23 Plummer M. JAGS: A program for analysis of Bayesian models using Gibbs sampling. In: Proceedings of the 3rd International Workshop on Distributed Statistical Computing; Vienna, Austria. 2003.

24 Viechtbauer W. Conducting meta-analyses in R with the metafor. J Stat Softw. 2010;36:1-48. doi:10.18637/jss.v036.i03

25 Hedges LV, Vevea JL. Fixed- and random-effects models in meta-analysis. Psychol Methods. 1998;3:486-504. doi:10.1037/1082-989X.3.4.486

26 Sutton AJ, Abrams KR, Jones DR, Jones DR, Sheldon TA, Song F. Methods for meta-analysis in medical research. Vol. 348. Chichester: Wiley; 2000.

27 Higgins JPT, Thompson SG, Deeks JJ, Altman DG. Measuring inconsistency in meta-analyses. BMJ. 2003;327:557. Medline:12958120 doi:10.1136/bmj.327.7414.557

28 Deeks JJ, Higgins JP, Altman DG. Analysing Data and Undertaking Meta-Analyses. In: Cochrane Handbook for Systematic Reviews of Interventions: Cochrane Book Series. London: Cochrane; 2008.

29 United Nations. World Population Prospects: The 2019 Revision. New York: United Nations; 2019.

30 Ks S, Jotheeswaran AT, Girish N, Bharath S, Dias A, Pattabiraman M, et al. The Dementia India report: prevalence, impact, costs and services for Dementia: executive summary. Available: http://ardsi.org/downloads/ExecutiveSummary.pdf. Accessed: 28 September 2019.

31 World Bank. GDP (current US\$) - Nepal. Washington, DC: World Bank; 2019. 